\title{
RAPPEL DES TRAVAUX DU COMITÉ TECHNIQUE DE LA SOCIÉTÉ HYDROTECHNIQUE DE FRANCE DEPUIS SA FONDATION
}

Les références des publications de ces travaux ou de leurs résumés, déjò parus, sont données à la suite du nom de l'auteur et du titre, suivant les abréviations ci-après :

- P.V.S. ......

Procès-verbal de la séance du... (date de la séance). A. P.V.S. .....

Annexe au procès-verbal de la séance du.... (date de la séance):

- C.R. $n^{\circ}$

Publication spéciale de la S.H.F., compte rendu $n^{\circ}$. - C. R. A. 19 .

Compte rendu d'activité de la S.H.F., année 19...

- R. G. E.

Revue Générale de l'Electricité.

R.G.H.

Revue Générale de l'Hydraulique.

Les mémoires ou lés résumés de ces travaux peuvent être consu'tés au Secrétariat de la Sociéte Hydrotechnique de France, 199, rue de Grenelle, a Paris $\left(7^{\circ}\right)$, qui, en outre, peut frurnir sur demande :

- les publications mentionnées \&. C.R. » ou «C.R.A.»

- ces photostats, micro-films ou analyses de tous les travaux.

\section{MYDRAULIQUE FLUYIALE ET MARITIME}

BOUCHER

Dessob!ement des eaux courantes:

P. V. S. 21-1-1922

R. G E $n^{\circ} 18,15-7-1922, p, 229 \mathrm{H}$.

S. H. F. (Commission des Débits solides)

Enquête sur les effets des barrages sur le lit des cours

d'eau et I'él mination des matériaux solides.

R. G. E. $n^{\circ} 24,10-5-1924$, p. $253 \mathrm{H}$.

V.-M. HEGLY

Note sur la répartition des vitesses dans un canal à pro.

fil complexe.

A. P. V. S. 14-2-1930

R. G. E. n' $43,1-11-1930$, p. $519 \mathrm{H}$.

V.-M. HEGLY

Note sur la répartition des vitesses dans un canal à pro-

fil comp'exe.

A. P. V.S. 30-6-1932

R. G. E. n 51, 23-9-1933; p. $655 \mathrm{H}$.

V.-M. HEGLY

Note sur l"app'ication à un grand fleuve des résultats d'expérience sur modèles réduits des canaux à profil comp'exe.

A. P. V. S. 9-11-1934.

R. G. H. n ${ }^{\circ} 55$, mars-avril 1935, p. $721 \mathrm{H}$

Ph. DEYMIE

Propacation d'une infumescence allongée.

A. P. V. S. 9-11-1934.

R. G. H. n 55 , mars-avril 1935, p. $730 \mathrm{H}$

\section{P. MASSÉ}

L'amortissement des intumescences.

A. P. V. S. 12-4-1935.

R. G. H. mai-juin 1935 p. $752 \mathrm{H}$.

P. MASSÉ

Le problèmé du Mascaret.

P. V. S. 28-2-1936.

R. G. H. $n^{0} 58$, mars-avril 1936, p. $792 \mathrm{H}$.

J. LAMOEN

Sur l'Hydraulique dec fleuves à marée.

A. P. V. S. 5-6-1936.

R. G. H. no 59 , nov.-déc. 1936, p. $815 \mathrm{H}$.

R. BOURGEAT

Erosions subies par un radier de pont

P. $\mathrm{S}$. $1-4-1938$

HEGLY

Onces produites dans un bief court par la manceuvre des vannes oux écluces d'extrémité

P. V. S. 8-11-1934

R. G. H. janv-février 1936, p. $763 \mathrm{H}$.

\section{COYNE}

Evacuateurs de Crues.

P. V.S 29-6-1934.

GARIEL

Partiteurs de débit.

P. V.S. 12-4-1935.

CAMICHEL

Les indéterminations et les solutions multiples dans leurs rapports avec I'hvdraulique fluviale.

P. V. S. 18-10-1935.

R. G. H. n 57, janv. fév. 1936, p. $762 \mathrm{H}$.

HEGLY

Influence d'une courbe dans le tracé d'un canal ò profil complexe au point de vue de la réportition des vitesses entre l'une et l'outre nartie du canal.

P. V.S. 18-10-1935.

R. G. H. n” 57, janv.-fév. 1936, p. $765 . \mathrm{H}$

MAUX

Conaux d'irrigation à marée en Indochine.

P. V. S. 28-2-1936.

R. G. H. n 58 , mars-avril 1936, p. $755 \mathrm{H}$. et $778 \mathrm{H}$

HEGLY

Construction de deux modèles réduits du barrage de Poses sur la Seine.

P. V. S. 28-2-1936

R. G. H. n58, mars-avril 1936, p. $77 \mathrm{H}$.

BESSON

Etude internationale sur la mesure des efforts de la mer.

- Appareils emplovés. - Résultats obtenus.

P. V. S. 5-6-1936.

R. G. H. no 59 , nov.-déc. 1936 , p. $809 \mathrm{H}$ 


\section{ESCANDE}

Piles à profil aérodynamique. - Cas des ouvrages construits au-dessus d'un déversoir.

P. V. S. 5-6-1936

R. G. H. no 59, nov,-déc. 1936, p. $810 \mathrm{H}$.

BARRILLON

Etude sur le passage de l'eau sous les murs de quai.

P. V. S. 5-6-1936.

R. G. H. n 59 , nov.-déc. 1936, p. $810 \mathrm{H}$

\section{COYNE}

Energie de la houle. - Déversoir de l'usine de Marègeș. P. V. S. 5-6-1936.

R. G. H. n 59 , nov -déc 1936, p. $814 \mathrm{H}$

VILLAIN

Etude des cuvrages à la mer.

P. V.S. 5-11-1936.

R. G. H. n 60 , mars-avril 1937, p. $845 \mathrm{H}$.

Mlle Francoise MILON

Etude systématique des températures du lac d'Annecy.

P. V. S. 5-11-1936

R. G. H. n 60 , mars-ovril 1937, p. $848 \mathrm{H}$

HEGLY

Quelques particularités de l'écoulement en aval du modèle du barrage dé Poses.

P. V. S. 5-11-1936.

R. G. H. n' 60 , mars-ayril 1937, p. $848 \mathrm{H}$

TOURNIER

Entretien et amélioration des chenaux par l'emploi d'un nouveau mode opératoire dit «fluxage».

P. V. S. 5-11-1936.

R. G. H. n' 60 , mars-avril 1937, p. $849 \mathrm{H}$.

MASSE

Sur le ressaut et la ligne d'eau dans les cours d'eau d pente variable.

P. V. S. 9-7-1937.

R. G. H. n 62 , sept.-oct. 1937, p. $914 \mathrm{H}$ (texte in extenso : R. G. H. n 19, janv.-févr. 1938, p. 7): MASSE

Des intumescences dans le's torrents.

P. V. S. 9-7-1937.

R. G. H. no 62 , sept.-oct. 1937, p. $915 \mathrm{H}$. (texte in ex. tenso R. G. H. $n^{\circ} \cdot 19$ et 20 , année 1938 , p. 7 et 61 ). BARRILLON

Les mouvements de seiche dans une baie

P. V. S. 11-3-1937.

Texte in extenso R. G. H. n 19, janv.-fév. 1938, p. 3. CAGNIARD

Propagation des intumescences vers l'amont ou l'aval des rivières

P. V.S. $11-3-1937$

GOURRET

Certains mouvements périodiques de la mer au voisinage d'une paroi oblique ou courbe.

P. V. S. 5-11-1937.

R. G. H. mai-juin 1938, p. 145 .

\section{LIMOUSIN}

Sur les essais en modèle réduit dans le domaine de I'hydroulique fluviale.

P. V. S. 5-11-1937

R. G. H. mai-juin 1938, p. 147 (texte in extenso R. G. H. $n^{\text {ss }} 21$ et 22,1938 ).
HENRY

Propagation des intumescences dans un canal rectangulaire.

P. V.S. 5-11-1937

R. G. H. mai-juin 1938 , p. 148 (texte in extenso R. G H. $n^{0} .19$, janv.-fév. 1938, p. 17).

COUTEAUD

Le résultat des essais sur modèle réduit relatifs à l'entrée du port Edouard-Herriot.

P. V. S. 26-10-1938.

R. G. H. mai-juin 1939, p. 151 .

\section{BOURGEAT}

Expériences sur la propagation des intumescences.

P. V.S. 26-10-1938.

R. G. H. mai-juin 1939 , p. 154 .

GLANGEAUD

Débit solide et dépôt dans les estuaires

P. V.S. 12-5-1939.

R. G. H. nov.-déc. 1939, p. 324

LE BRUN

Colcul pratioue des remous

C. R. A. 1943.

GAGE

Les galeries de dérivation proviscire

P. V.S. 4-2-1943

MICHE

Les mouvements ondulatoires de la mer et la forme limite ce la houle lors du déferlement.

P. V.S. 17-7-1943.

R. G. H. n 32 , p. 51 .

\section{REMENIERAS}

Note sur la modification de la chambre de mise en charge de l'usine I du Saut-Mortier.

P. V.S. $15-2-1944$.

MONTAGNE

Procédé et dispositif d'aménagement de cours d'eau formant dessableur.

C.R. A. 1945.

MONTAGNÉ

Localisation des pertes du tunnel en charge de l'usine

d'Eylie (Arièqe)

C. R. A. 1945

M. COQUANT

Mode d'action de certains épis en rivière.

C. R. A. 1945.

M. MICHE

Quelques observations de $M$. Darras sur son étude «Mouvements ondulatoires de la mer en profondeur constante ou décroissante $\%$

C. R. A. 1945:

LABAYE

Débit solide en canaux et rivières : quelques vues sur des recherches à entreprendre à propos du charriage de fond. C. R. A. 1945

P. JONTE

Eturte des coupures des boucles de l'Isère en amont de Grenoble.

P. V.S. $19-11-1946$.

Mémoires et Travaux $n^{\circ} 1-1949, p, 46$.

LARRAS

Comment prévoir les déformations d'une houle donnée arrivant dans une région où les fonds sont variables et où les ouvrages peuvent gêner, sa propogation.

P. V.S. Sous-Commission 21-2-1947. 


\section{VALEMBOIS}

Energie de la houle ou large et près du rivage.

P. V. S. Sous-Commission 21-2-47

\section{ESSAIS ET MESURES}

\section{HAHN}

Etude sur l'emploi de's déversoirs triangulaires.

P.V.S. $19-12-1912$

\section{RATEAU}

Jaugeage par tuyères noyées.

P. V. S. 26-6-1913.

S. H.F.

Règlement sur l'exécution des essais. - Règlement intérieur.

F.V.S. 18-9-1913.

A. BOUCHAYER

Notes sur des expériences d'Hydraulique

P. V.S. 20-9-1913

CAMICHEL et EYDOUX

Emploi du jaugeur Venturi.

P. V.S. $2-4-1947$

La Lumière Electrique, Tome XXVI, 1-8-1914.

C. HERSCHEL

Noté sur un nouvequ type de déversoir.

P. V.S. $24-6-1920$

GARIEL

Notes sur les essais de pertes de charge que l'on pour rait faire sur les conduites forcées de Soulom et d'Eget

P. V.S. $25-11-1920$.

R. G. E. n $n^{\circ} 13,21-5-1921,0.147 \mathrm{H}$

BARBILLION

Méthode thermométrique pour mesurer le rendement des turbines.
P.V.S. $3-9-1921$

R. G. E. $n^{\circ} 16,29-4-1922$, p. 203 H., n 17, 27-5-1922, p. 227 H. et $n^{\circ} 19,16-9-1922$, p. $233 \mathrm{H}$.

BOURGEAT

Etude de déversoirs en mince paroi pour de faibles charges:

A. P. V.S. 12-4-1924.

R. G. E. n $26,9-8-1924$, p. $271 \mathrm{H}$

COMITE TECHNIQUE S.H.F.

Pertes de charge dans une conduite rivée de $300 \mathrm{~mm}$ de diamètre intérieur et $7 \mathrm{~mm}$. d'épaisseur.

A. P. V. S. 12-4-1924

R. G.E. $n^{\circ} 27,13-9-1924$, p. $275 \mathrm{H}$.

COMITÉ TECHNIQUE S, H. F. (BOURGEAT).

Pertes de charge dans une conduite lisse de $300 \mathrm{~mm}$. de diomètre intérieur.

A. P. V. S. $20-2-1924$

R. G. E. $n^{2} 29,6-6-1925$, p. $287 \mathrm{H}$.

COMITE TECHNIQUE S. H.F. (BOURGEAT).

Expériences sur les pertes de charge faites à létranger. A. P. V.S. $13-5-1925$

R. G. E. $n^{\circ} \times 30,17-10-1925$, p. $295 \mathrm{H}$.

Jean LAURENT

Organisation du Service de contrôle permanent des installations hydroélectriques. - Nouveaux appareils employés par le Service des Essais extérieurs de la S.H.F.

A.P. V.S. 19-2-1926.

R. G. E. $n^{\circ} 33,12-6-1926$, p. $327 \mathrm{H}$

\section{R. BOURGEAT}

Influence de la tempéroture de l'eau sur la perte de charge dans les condutes circulaires.

A.P.V.S. 2-11-1926.

R. G.E. n. $35,40-4-1927$, p. $346 . \mathrm{H}$.

P. LEROUX

Détermination du coefficient dé débit des tuyères fonctionnant en écoulement libre

A.P. Y. S. 2-11-1927

R. G. E. n". 37, 22-10-1927, p. $380 \mathrm{H}$

P. LEROUX ET BOURGEAT

Station d'étalonnage en eau courante d'appareils de mesures hydrométriques du 'Laboratoire de lo Société Hydrotechnique dé France à Beauvert (Grenoble).

A. P. V. S. 19-1 1-1927.

R. G. E. n" 38, 28-4-1928, p. $387 \mathrm{H}$

BOULLE ET A. LANGEVIN

Etude des coups de bélier dans les canalisations de faibles longueurs et de petits diamètres ò l'aide d'un enre. gistreur piézoélectrique à lame de quartz.

A. P. V. S: $14-2-30$.

R. G. E. $n^{6} 42,16-8-1930$, p. $507 \mathrm{H}$.

A. FOCH et J: LAURENT

Note sur une difficulté présentée par les mesures au moulinet.

A. P. V.S. $14-2-30$

R. G. E. $n^{\prime} 43,1-11-1930$, p. $531 \% \mathrm{H}$

R. BOURGEAT

Evaluation du débit des canalisations par l'insertion de tuyeres ou orifices.

A. P. V.S. 24-2-1933

R. G. E. $\mathrm{n}^{\circ} 52,23-12-1933$, p. $689 \mathrm{H}$

P. DELATTRE Et P. LEROUX

Essai d'un modèle réduit de déversoir.

R. G. E. n $n^{\circ} 53$ du 22-9-34, p. $697 \mathrm{H}$.

BOURGEAT, D. CAHUZAC et J. DEULLIN

Essais de cheminées d'équilibre sur modèles réduits.

A. P. V. S: $12-4-35$.

R. G. H. mai-juin 1935, p. $742 \mathrm{H}$.

R. BOURGEAT

L'étalonnage des moulinets au Laboratoire de Beauvert.

A. P. V.S. 5-6-1936

R. G. H. n $n^{\circ} 58$, mars-avril 1946, p. $806 \mathrm{H}$

R. BOURGEAT, D. CAHUZAC et J. DEULLIN

Recherches expérimentales. sur l'étalonnage des moulinets en eau calme.

A. P. V.S. $5-11-1936$

R.G.H. $n^{\circ} 60$, mars-avril 1937, p. $873 \mathrm{H}$.

André LANGEVIN

Utilisation de l'effet piézo-électrique du quartz pour

l'érude des coups dé bélier dans les conduites forcées.

A. P. V. S. 9-7-1937.

- R. G. H. n. 62, sept.oct. 1937, p. $913 \mathrm{H}$.

R. BOURGEAT e't L. NETTER

Essais sur modèle réduit d'une vanne secteur.

A.P. V.S. $9-7-1937$

R. G. H. no 62, sept.oct. 1937 , p. 939 H.

C. R, $n^{\circ} 62$.

P. BESSON

Les appareils de mesure des efforts des lames

C. R. $n^{*} 62$

A. P.V.S. $9-7-1937$

R. G. $H, n^{\circ} 62$, sept.oct. $1937, \mathrm{p}, 949 \mathrm{H}$ 
M. BOUCHER

Manomètre différentiel.

P. V. S. 20-3-1931.

R. G. E. $n^{\circ} 46$, p. $556 \mathrm{H}$.

Jean LAURENT

Influence des coudes sur les venturi.

P. V. S. 20-3-1931.

R. G. E. $n^{\circ} 46$, p. $566 \mathrm{H}$.

Jean LAURENT

Expériences du Laboratoire de Walchensee sur la comparaison des différentes méthodes de mesure des débits.

P. V. S. 20-3-1931.

R. G.E. $n^{\circ} 46$, p. $566 \mathrm{H}$.

R. BOURGEAT

Au sujet d'une note de $M$. Pers sur les stéréo et vectopluviomètres.

P. V. S. 8-11-1934

\section{EYDOUX}

Dispositif de $M$. Ledoux pour la mesure de variations de nivecu infiniment petites.

P. V. S. 12-4-1935.

R. G. H. $n^{\circ} 56$, p. $379 \mathrm{H}$.

HAHN latérale.

Etude sur le déversoir en mince paroi sans contraction

P. V. S. 18-10-1935.

R. G. H. janv.-fév. 1936, p. $762 \mathrm{H}$

Jean LAURENT

Mesure des pertes de charge dans la petite robinet terie.

P. V. S. 28-2-1936

R. G. H. $n^{\circ} 58$, mars-avril 1936 , p. $77 \mathrm{H}$.

Jean LAURENT

Sur le congrès d'Edimbourg de l'Association Interna. tionale d'Hydrologie scientifique : Organisation d'une enquête internationale sur la précision des mesures hydrauliques).

P. V.S. $5-11-1936$

R. G. H. $n^{\circ} 60$, mars-avril 1937 , p. $851 \mathrm{H}$.

AURIC

Graphique présentant les résultats d'essais d'appareils hydromécaniques.

P. V. S. 9-7-1937

R. G. H. sept.-oct. 1937, $n^{\circ} 62$, p. $913 \mathrm{H}$.

COT houle.

Description d'un nouvel appareil pour la mesure de la

P. V. S. $11-3-1937$.

PETRY

Pressions et vitesses dans les lames déferlantes.

P. V.S. 5-11-1937.

R. G. H. mai-juin 1938 , p. 146.

L. BERGERON

Etat actuel du projet d'étude expérimentale des coups de bélier.

P. V.S. 1-4-1938.

R. G. H. nov.-déc. 1938 , p. 324.

MASON

Contribution à l'étude de la mesure des débits d'eau par la méthode d'Allen.

P. V. S. 26-10-1938.

R. G. H., mai-iuin 1939, p. 149 (texte in extenso :

R. G. H. no 28, juillet-août 1939, pp. 177-194 et

$n^{\circ} 29$, sept.-oct. 1939, pp. 242-259.
DUCHENE

Sur une méthode de mesure des débits gazeux

P. V. S. 26-10-1938.

R. G. H. mai-juin 1939, p. 149.

BOURGEAT (Travaux du Laboratoire de Beauvert)

Tarage de canaux.

P. V. S. 26-10-1938.

R. G. H. mai-juin 1939, p. 155.

BOURGEAT (Travoux du Laboratoire de Beauvert

Expériences sur le tarage des moulinets.

P. V. S. 26-10-1938.

R. G. H. mai-juin 1939, p. 156.

HEGLY (Travaux du Laboratoire du Saulcy)

Essais sur modèles réduits de revêtement d'un canal.

P. V. S. 26-10-1938.

R. G. H. mai-juin 1939, p. 157.

HEGLY (Travaux du Laboratoire du Saulcy)

Expériences sur le tarage des moulinets.

P. V. S, 26-10-1938.

R. G, H. mai-juin 1939 , p. 157

HEGLY (Travaux du Laboratoire du Saulcy)

Expériences sur les ondes de translation en canaux découverts (rigole ce Valcourt).

P. V. S. 26-10-1938

R. G. H. mai-juin 1939, p. 157

BOURGEAT (Travaux du Laboratoire de Beouvert)

Expériences sur les intumescences

P. V. S. 12-5-1939.

R. G. H. nov.-déc. 1939 , p. 328 .

BOURGEAT (Travaux du Laboratoire de Beauvert)

Expériences sur la viscosité de's huiles destinées au graissage des moulinets.

P. V. S. $12-5-1939$

R. G. H. nov.-déc. 1939 , p. 329.

COLLET

Théorie d'un ampteur alcoométrique.

P. V. S. 25-5-1945.

J. VALEMBOIS

Recherches théoriaues et expérimentales sur la mesure des pressions fluctuantes.

P. V. S. 19-11-1946.

J. VALEMBOIS

Même sulet : La Mesure des pressions variables. Hermann, éditeur, Paris, 1948.

\section{MACHINES HYDRAULIQUES}

S. H. F:

Cahier des charges pour marchés de turbines.

P. V. S. 2-6-1914.

DE SPARRE

Remarque au sujet du degré de réaction à admettre pour les turbines dans le but a'augmenter leur vitesse de rotation.

P. V. S. $4-2-1919$.

R. G. E. $n^{\circ} 11,13-11-1920$.

DE SPARRE

Note au sujet des Turbines centripète's à réaction.

P. V. S. 25-11-1920.

R. G. E. n $n^{\circ} 13,21-5-1921$, p. $148 \mathrm{H}$. 


\section{DE SPARRE}

Calcul des coups de bélier dans les conduites desservant des tubbines à forte réaction.
P. V. S. $3-9-1921$.
R. G. E. n $16,29-4-1922$, p. $205 \mathrm{H}$

DE SPARRE bines.

Remarque au sujet du colcul du rendement des tur-

P. V.S. $3-9-21$.

R. G. E. n $n^{\circ} 16,29-4-1922$, p. $215 \mathrm{H}$

HAHN

Résumé de l'étude présentée au congrès de Liége sur le tracé des aubes à grance vitesse

P. V.S. 26-4-1923.

R. G. E. $n^{\circ} 22,14-7-1923$, p. $246 \mathrm{H}$

EYDOUX

Les bases hycrodynamiques de la theorie des turbomachines hydrauliques.

A. P. V. S. 13-5-1925.

R. G. E. $n^{6} 31,24-10-1925$, p. $301 \mathrm{H}$

E. DUSAUGEY

Note sur les avantages et les inconvénients comparés des groupes hydroélectriques à axe vertical et à axe horizontal.

A. P. V. S. $19-11-1927$

R. G. E n $38,28-4-1928, p .395 \mathrm{H}$

A. METRAL

Corrosions et cavitations dans les turbo-machines hydrauliques.

A. P. V: S. $11-5-1928$

R. G. E. $n^{\circ} 39,30-6-1928$, p. $403 \mathrm{H}$

P. LEROUX

A. P. V S 24-2-1933

Résumé sur l'état actuel de la question des diffuseurs

R. $G, E$ n ${ }^{\circ} 52,23-12-1935$, p. $678 \mathrm{H}$ GARIEL

Turbines tourbillons Reiffenstein.

P. V. S. $8-11-1934$

BERGERON

Influence de l'état tourbillonnaire de l'eau sur le fonctionnement d'une pompe.

P. V.S. $12-4-1935$.

HAHN

Détermination des angles d'entrée et de sortie des aubes de distributeurs et roues motrices des turbo-machi nes.

P. V. S. $18-10-1935$

R. G. H. no.57, janv,-fév. 1936, p. $764 \mathrm{H}$

COLLET

Obturateurs automatiques pour conduites d'eau

P. S. 18-10-1935

R. G. H. n 57 , jany -fév. 1936, p. $764 \mathrm{H}$

J. LAURENT

L'Usure dons les turbines et ses conséquences sur le renc'ement

P. V.S. 5-6-1936.

R. G. H. n 59, nov-déc. 1936, p. $811 \mathrm{H}$

DUPUIS

Lo détermination des trajectoires des particules d'eau dans l'écoulement ò travers une pompe centrifuge.

P. V. S. 5-11-1937.

R. G. H. mai-juin 1938, p. 149.
VADOT

Etude expérimentale de l'écoulement méridien dans les turbines.

P. V. S. $26-10-1938$

R. G. H. mai-juin 1939, D. 154 (texte in extenso :

R. G. H. n 28, juillet-août 1939 , pp. 203 à 207).

CHENAIS

Travaux de la Commission d'étude de la limite de puissance des machiners hyćrauliques.

P.. V. S. 2-6-1944.

P. V. S. Section 10-2-1944.

\section{CHENAIS}

Modèle réduit de groupe Turbine-Alternateur destiné à l'étude de la stabilité de groupes hydroélectriques interconnectés. - Régulation des groupes records.

A. P. V. S. Section 10-2-1944.

\section{GADEN}

Lettre à M. Bergeron, président de la S.H.F. sur la stabilité de réglage

P. V. S Section 2I-1-1944

L. BERGERON

Limitation des puissances des machines hydrauliques.

P. V. S. 2-6-1944.

\section{GENIE CIVIL ET CONDUITES}

S. H. F

Marché et Cahier des charges pour la fourniture des conduites forcées en béton de ciment armé

P. V. S. Conseil d'Adm. 24-1-1920

R. G. E. $n^{\circ} 6,13-3-1920$, p. $73 \mathrm{H}$. (en cours de revision).

G. MERCIER, GENTHIAL

Conférence à l'Ecole Nationale des Ponts et Chaussées sur le congrès des Gands Barrages de Washington de 1936

P. V.S. 5-11-1936.

R. G. H. n 60 , mars-avril 1937, p. $852 \mathrm{H}$

J. $\angle A U R E N T$

Sur les mesures de déformation les barrages.

P. V.S. 1-4-1938.

R. G. H. nov.-déc. 1938 , p. 324 .

COYNE

Le Nil et le Barrage d'Assouan.

P. V.S. 1-4-1938.

R. G. H. nov.-déc. 1938 , p. 327 .

COYNE

Barrage ce St-Michel.

P. V. S. $1-4-1938$.

R. G. H. nov.-déc 1938 , p. 328 .

WAEBER

Travaux de la prise d'eau sous-glaciaire réalisée au glacier de Tré-la-Tête.

P. V. S. 20-12-1945.

Annales des Ponts et Chaussées, juillet-août 1945.

\section{GENTHIAL}

Application du règlement S. H.F. concernant les téléfériques aux chantiers d'équipement hydroélectrique en cours.

P. V.S. 29-4-1947 


\section{GENERALITES ET ETUDES DIVERSES}

\section{PEROT}

Note sur les conditions à remplir dans la substitution de la houille blanche à la houille noire.

P. V. S. 15-12-1916.

\section{BACHELLERY}

Note sur le transport de l'énergie électrique aux grandes distances.

P. V. S. 5-12-1918.

R. G. E. $n^{\circ} 9,10-7-1920, p, 113 \mathrm{H}$

C. R. $n^{\circ} 9$

\section{AUBRY}

Note sur les conditions pratiques dans lesquelles sont actuellement réalisées les jonctions de réseaux de distribution.

P. V. S. $3-9-1921$.

R. G. E. n $n^{\circ} 14$, p. $169 \mathrm{H}$.

\section{BERGEON}

Chaudières électriques

P. V. S. 8-6-1922.

R. G. E. $n^{0} 19,16-9-1922$, D. $234 H$

MARY

Aménagements hydrauliques de l'usine de Marèges.
P. V. S. $9-11-1934$.

R. G. H. n 55 , mars-avril 1935, p. $723 \cdot \mathrm{H}$.

\section{AUBERT}

L'aménagement du Rhône (Conférence à l'Ecole Nationale des Ponts et Chaussées).

P. V. S. 5-11-1937.

R. G. H. mai-juin 1938 , p. 149.

L. BERGERON

Note sur mon voyage en Amérique

P. V. S. $1-4-1938$.

R. G. H. nov.-déc 1938 , p. 323 .

L. BERGERON

Sur le problème de la chute d'une masse sur un prisme rectiligne élastique, traité par Boussinesq et Saint-VEnant

P. V. S. 26-10-1938.

R. G. H. mai-juin 1939, p. 153.

TENOT

Frein hydraulique dynamométrique système « Tenot Pont-à-Mousson». - Son adaptation aux grandes puissances.

C. R. A. 1945.

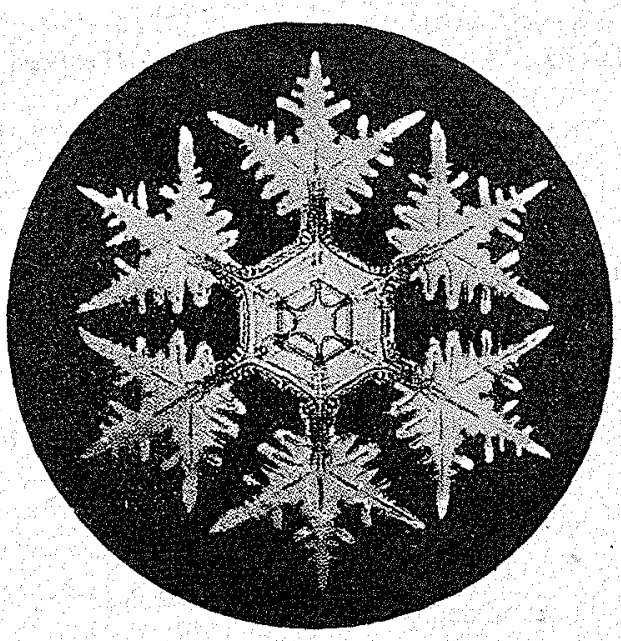

